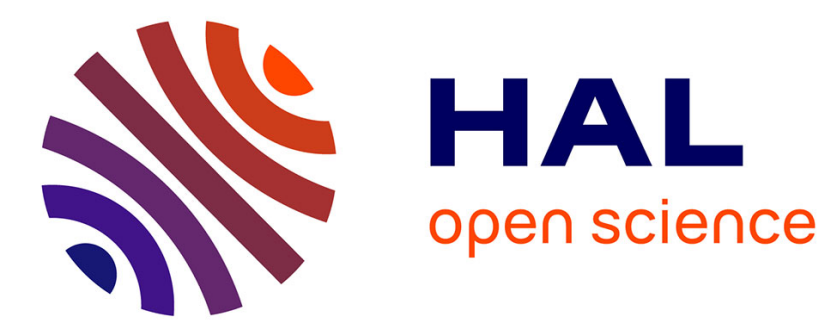

\title{
Game of Trans-Kingdom Effectors
}

Sophie Bleves

\section{- To cite this version:}

Sophie Bleves. Game of Trans-Kingdom Effectors. Trends in Microbiology, 2016, 24 (10), pp.773 774. 10.1016/j.tim.2016.08.002 . hal-01766104

\section{HAL Id: hal-01766104 https://hal-amu.archives-ouvertes.fr/hal-01766104}

Submitted on 13 Apr 2018

HAL is a multi-disciplinary open access archive for the deposit and dissemination of scientific research documents, whether they are published or not. The documents may come from teaching and research institutions in France or abroad, or from public or private research centers.
L'archive ouverte pluridisciplinaire HAL, est destinée au dépôt et à la diffusion de documents scientifiques de niveau recherche, publiés ou non, émanant des établissements d'enseignement et de recherche français ou étrangers, des laboratoires publics ou privés. 
1 Title

\section{Game of trans-kingdom effectors}

3

4

5 Sophie Bleves*

6

7

8 Aix-Marseille Univ, CNRS, LISM, Laboratoire d'Ingénierie des Systèmes

9 Macromoléculaires-UMR7255, IMM (Institut de Microbiologie de la Méditerranée),

10 Marseille, France.

$11 *$ *PseudomonasCNRS

12

13

14 Correspondence: bleves@imm.cnrs.fr (S. Bleves)

15

16 
Keywords

Type VI secretion system, Pseudomonas aeruginosa, trans-kingdom effector, antibacterial, endoplasmic reticulum stress, autophagy

Abstract

TplE, a type VI secreted (phospho)lipase, has been identified as the third transkingdom effector of Pseudomonas aeruginosa, targeting both prokaryotic and eukaryotic hosts. Indeed, TplE triggers killing of bacterial competitors and promotes autophagy in epithelial cells once localized to the endoplasmic reticulum.

\section{Main text}

The type VI secretion systems (T6SSs) are present in more than $25 \%$ of Gramnegative bacteria, making T6SS one of the most widespread protein secretion systems [1]. It functions as a molecular crossbow that mainly delivers toxic effectors into bacterial target cells to antagonize and eliminate competitors in close proximity [1]. Besides the benefits in the environmental habitat, bacteria can use T6SS in the context of eukaryotic host by two divergent routes [1]. On one hand, T6SS can directly target eukaryotic cells through delivery of effectors in their cytosol, highjacking host cellular pathways or triggering toxicity. On the other hand, pathogens which are in close contact with the commensal microbiota while infecting a host, can attack or resist T6SS-dependent attack from commensal microbiota, in order to colonize and/or persist within the host $[2,3]$. Altogether, T6SSs are key new players in the host-pathogen-microbiota complex interactions, through both anti-eukaryotic and antibacterial activities. 
T6SS has been the first secretion system shown to mediate interactions with prokaryotic and eukaryotic hosts through dedicated and diverse toxins [1]. The case of Pseudomonas aeruginosa is unique in this trait since three T6SS-dependent effectors are capable to target both types of host cells making them the first examples of trans-kingdom effectors [4,5]. Besides being responsible for chronic lung colonization of patients with cystic fibrosis, $P$. aeruginosa is one of the leading causes of hospital-acquired infections. Its genome encodes three independent T6SSs. H1-T6SS is so far exclusively dedicated to antibacterial activity through injection of at least 7 different effectors, whereas H2-T6SS and H3-T6SS have a dual role allowing targeting of both prokaryotic and eukaryotic cells [6]. Even considered an extracellular pathogen, $P$. aeruginosa is able to actively invade non-phagocytic cells, through injection of effectors PldA and VgrG2b via H2-T6SS [4,7], and PldB via H3T6SS [4] among other factors. On the one hand, PldA and PldB bind the Akt kinase, whose activation together with the recruitment of the PI3K (phosphoinositide 3-kinase) pathway is further required for actin polymerization at the bacterial binding site leading to bacterial invasion [4]. On the other hand, VgrG2b promotes a microtubule-dependent internalization of $P$. aeruginosa through interaction with the gamma-tubulin complex, demonstrating the spectacular ability of $P$. aeruginosa T6SSs to highjack host cellular functions to its own advantage [7].

In a recent report from Jiang and colleagues, TplE was identified as the third T6SS trans-kingdom effector of P. aeruginosa [5]. Such as PldA and PldB, TplE (formerly called Tle4) belongs to the large antibacterial type VI lipase effector family (Tle) [8]. In agreement with this, its structure exhibits the characteristics of a canonical $\alpha / \beta$-hydrolase fold [9]. Interestingly, Jiang and colleagues [5] demonstrated that TplE harbors lipase and phospholipase A1 activities in vitro. The authors first confirmed that TplE mediates antibacterial activity, by producing $\mathrm{TplE}$ and a serine catalytic variant in the periplasm of 
Escherichia coli. Importantly, only wild-type TplE led to bacterial growth inhibition, while the catalytic variant of TplE was not toxic. As previously proposed [8], and consistent with a crab claw-like conformation of TplEi (formerly Tli4) [9], the product of the downstream gene TplEi (TplE immunity), neutralized the TplE toxicity. Indeed, to protect from self or sister cell killing, bacteria produce immunity proteins to counteract their cognate T6SS antibacterial effectors. Authors further demonstrated that TplE lipolytic activity contributes to intra- and inter-species competition once delivered into target bacteria via the H2-T6SS machinery and that TplEi provides protection against TplE.

Furthermore, the role of TplE during interaction with eukaryotic target cells was also characterized in this report, by demonstrating the H2-T6SS-dependent translocation of TplE into epithelial cells upon infection with $P$. aeruginosa [5]. Interestingly, TplE contains a eukaryotic PGAP1 (Post GPI Attachment to Proteins 1)-like domain present in proteins localized at endoplasmic reticulum. The targeting of TplE to this compartment was further demonstrated with a combination of ex vivo assays (transfection and infection). One can note that the enzymatic activity of TplE was not required for endoplasmic reticulum localization. Nevertheless this targeting led to endoplasmic reticulum disruption, which in this case, involves the lipolytic activity of TplE. The authors also observed that in response to this endoplasmic reticulum stress, TplE activated the unfolded protein response via the XBP1 pathway and triggered autophagy, as shown by LC3 conversion and the increase of autophagosomes and autolysosomes. The integrity of the TplE active site was determinant for these two processes. Autophagy is a conserved destructive process allowing degradation of unnecessary, dysfunctional, or undesirable cellular components including organelles (like the endoplasmic reticulum) or intracellular pathogens. In the latter case, autophagy is considered an innate immunity defense mechanism of infected cells against invading bacteria, but intracellular pathogens have evolved mechanisms modulating this host response to their 
14

advantage. The autophagy response activated by $P$. aeruginosa highlights the transient but real intracellular lifestyle of this pathogen, still being largely considered an extracellular pathogen.

Whereas the competitive advantage towards other bacteria conferred by TplE, PldA and PldB is quite simple, how could their actions towards eukaryotic cells be integrated in a common model? PldA, PldB and VgrG2b contribute to P. aeruginosa internalization into epithelial cells that can be view as a strategy to avoid innate immunity. However once intracellular, the destiny of $P$. aeruginosa is still unclear. The pathogen may exploit and benefit the autophagy to acquire nutriments from endoplasmic reticulum degradation and thus promote its own intracellular replication such as Francisella tularensis [10]. Consistent with this hypothesis, the H2-T6SS is not cytotoxic towards epithelial cells [4,7]. The use of autophagy modulators may be thus envisioned as a new therapeutic strategy in the fight against $P$. aeruginosa infections. The findings of Jiang and colleagues [5] provide new and important insights in the pathogenesis of $P$. aeruginosa that relies in bacterial competitor as well as in eukaryotic cells in a game of trans-kingdom effectors. Moreover since TplE, PldA and PldB belong to the superfamily of type VI lipase effectors present in various pathogens [8], one can ask whether other trans-kingdom effectors may be discovered in the future.

\section{Acknowledgements}

I would like to thank C. Soscia, my (former) PhD students (Richard, Thibault, Aurélie, Maxime and Benjamin), B. Ize, people from the LISM for constant support and Thibault Sana for reading of the manuscript. I acknowledge funding through the CNRS and Aix-Marseille Univ, Vaincre La Mucoviscidose and Fondation Grégory Lemarchal (RF20150501346).

\section{Reference list}


1161 Cianfanelli, F.R. et al. (2016) Aim, Load, Fire: The Type VI Secretion System, a bacterial 117 nanoweapon. Trends Microbiol. 24(1):51-62

1182 Fu, Y. et al. (2013) Tn-Seq analysis of Vibrio cholerae intestinal colonization reveals a role

119 for T6SS-mediated antibacterial activity in the host. Cell Host Microbe. 14,652-663

1203 Sana, T.G. et al. (2016) Salmonella Typhimurium utilizes a T6SS-mediated antibacterial 121 weapon to establish in the host gut. Proc Natl Acad Sci U S A. Published ahead of print $122 \quad$ August 5, 2016

1234 Jiang, F. et al. (2014) A single Pseudomonas aeruginosa type VI secretion phospholipase D

124 effector targets both prokaryotic and eukaryotic cells. Cell Host Microbe 15(5):600-10

1255 Jiang, F. et al. (2016) The Pseudomonas aeruginosa type VI Secretion PGAP1-like effector 126 induces host autophagy by activating endoplasmic reticulum stress. Cell Rep. S2211127 1247(16)30898-1

1286 Sana, T.G et al. (2016) The T6SSs of Pseudomonas aeruginosa strain PAO1 and their 129 effectors: beyond bacterial-cell targeting. Front Cell Infect Microbiol. 6:61

1307 Sana, T.G. et al. (2015) Internalization of Pseudomonas aeruginosa strain PAO1 into 131 epithelial cells is promoted by interaction of a T6SS effector with the microtubule network 132 MBio 6(3):e00712

1338 Russel, A.B. et al. (2013) Diverse type VI secretion phospholipases are functionally plastic 134 antibacterial effectors. Nature 496(7446):508-12

$1359 \mathrm{Lu}$, D. et al. (2014) The structural basis of the Tle4-Tli4 complex reveals the self-protection 136 mechanism of H2-T6SS in Pseudomonas aeruginosa. Acta Crystallogr D Biol Crystallogr. $137 \quad 70: 3233-43$

13810 Steele, S. et al. (2013) Francisella tularensis harvests nutrients derived via ATG5139 independent autophagy to support intracellular growth. PLoS Pathog 9(8): e1003562 